\title{
Erratum to: A possible evidence of observation of two mixed phases in nuclear collisions
}

\author{
K.A. Bugaev ${ }^{1}$, A.I. Ivanytskyi ${ }^{1}$, V.V. Sagun ${ }^{1}$, G.M. Zinovjev ${ }^{1}$, D.R. Oliinychenko ${ }^{1,2}$, V.S. Trubnikov ${ }^{3}$, \\ and E.G. Nikonov ${ }^{4}$ \\ ${ }^{1}$ Bogolyubov Institute for Theoretical Physics of the National Academy of Sciences of Ukraine, 03680 Kiev, Ukraine \\ ${ }^{2}$ FIAS, Goethe University, Ruth-Moufang Str. 1, 60438 Frankfurt upon Main, Germany \\ ${ }^{3}$ National Science Center "Kharkov Institute of Physics and Technology", 61108 Kharkov, Ukraine \\ ${ }^{4}$ Laboratory for Information Technologies, JINR, Joliot-Curie Str. 6, Dubna 141980, Russia
}

Original article: EPJ Web of Conferences 126, 03003 (2016), DOI: 10.1051/epjconf/201612603003

In caption of Fig. 3, it should have been indicated that this figure has been reproduced from [12] V.A. Kizka, V.S. Trubnikov, K.A. Bugaev and D.R. Oliinychenko, arXiv:1504.06483. 